\title{
Universal and Anisotropic Simulation Platform for the Study of Electrical Properties of Conductive Polymer Composites
}

\author{
Guanda Yang ${ }^{1}$, Muchao $\mathrm{Qu}^{1}$, Fritjof Nilsson ${ }^{2}$ and Dirk W. Schubert ${ }^{1}$ \\ 1. Institute of Polymer Materials, Friedrich-Alexander-University Erlangen-Nuremberg, Erlangen 91058, Germany \\ 2. KTH Royal Institute of Technology, School of Chemical Science and Engineering, Fibre and Polymer Technology, SE 10044 \\ Stockholm, Sweden
}

\begin{abstract}
In order to carry out a study on the synergistic relationship of variables which could affect the electrical properties of CPC, a universal and anisotropic platform of simulation, containing three simulation modules is explored. The simulation modules are: FEM (finite element modeling), PTM (percolation threshold modeling) and ENM (electrical networks modeling).
\end{abstract}

Key words: Conductive polymer composites, finite elements method, simulation.

\section{Introduction}

Due to easy fabrication, tunable properties and a broad range of applications, the research area of CPCs (conductive polymer composites), consisting of insulating polymers and conductive fillers (CB (carbon black), CFs (carbon fibers), graphene, etc.) has increasingly gained more attention in the last decade [1]. CPCs with good flexibility exhibit a variety of sensing responses when subjected to external strain. The sensing mechanism is mainly based on the destruction and reconstruction of conductive networks. Therefore a variation of conductivity can be linked to the amount of exposure by external stimuli.

Recently, CPCs with good flexibility have been considered as satisfactory candidates to develop flexible strain sensors. Utilizing the advantages of the polymer matrices, CPCs can show excellent stretching ability and quick response to external stress [2-4]. Elastomers, such as vinylidene

Corresponding author: GuandaYang, M.Sc., research fields: finite element simulation, data analysis, polymer composite, applied mathematics. fluoride-hexafluoropropylene copolymer [5], PDMS (polydimethylsiloxane) [6], SR (silicone rubber) [7-9] or TPU (thermoplastic polyurethane) [10-12] are typically used as polymer matrices. However, the application of CPCs based strain sensors still faces severe challenges, e.g., unstable signal output and insufficient reversibility. In particular, due to different demands for the strain sensing behavior in different applications, CPCs with tunable strain response patterns are urgently needed.

\section{Background}

Besides the experimental investigation, simulations can provide an effective way of understanding the dynamic mechanism [13-15]. A surplus of theoretical studies carried out by simulations has already been conducted in order to investigate the relationship between the geometry of fillers and electrical properties [16-18]. However, most of these studies assumed an idealized isotropic orientation of the fillers, represented by using just one parameter for the spatial orientation $[19,20]$. If the geometrical factors are considered, as for example the aspect ratios of the fillers and the filler inclination, the influence on the 
electrical properties can be studied thoroughly.

Besides the experimental investigation, simulation can provide an effective way of understanding the dynamic mechanism. Many excellent theoretical studies via simulations have already been conducted [1-3] in order to investigate the relationship between the geometry of fillers and electrical properties. However, most of these studies assumed an idealized isotropic orientation of the fillers, and focused on only one variable of geometrical distribution. Considering the geometrical factors, i.e. fillers' aspect ratios and filler inclination might influence the electrical properties [21, 22]. Therefore, a systematic theoretical study on the influence of the geometrical distributions and filler dimensionality, especially for the 3-dimensional fillers, would still be scientifically necessary.

\section{Model}

For the simulation and modeling, the influence of different parameters on the electrical conductivity, particularly on the electrical percolation filler fraction was discussed in relation to experimental data [23]. In order to carry out a study on the synergistic relationship of these variables, a universal and anisotropic platform of simulation, containing three simulation modules had been explored [24]. The simulation modules contain FEM (finite element modelling), PTM (percolation threshold modeling) and ENM (electrical networks modelling). After constantly updating, the simulation platform performs the following features:

- Anisotropy (orientation);

- High degree of flexibility;

- High degree of automation;

- High efficiency: Under the same magnitude of mesh and computing environment, our platform needs only 1/3 the time of other similar simulation program;

- High accuracy: All parameters and results can be accurate up to 12 decimal places;

- Very low error rate (less than $0.01 \%$ ).
The mesh strategy is the key of simulation. For simulation with different settings and conditions, it is necessary to bring different mesh strategy when facing diverse models. In the platform of simulating CPCs, the mesh strategy is always optimized and updated.

\section{Experimental}

The ordinate axis shows the number of elements, and the abscissa shows the ratio of current mesh quality and the smallest mesh quality [25]. For the 3D model, it could also be approximated as the reciprocal of the ratio of the minimum element volume to the current element volume. This distribution is a practical tool for judging the quality of the mesh and the authenticity of the simulation results. A curve that satisfies the normal distribution represents a suitable meshing.

Finite element modelling (FEM) in 3 dimensions was used in order to examine how the electrical conductivity of polymeric fiber composites is influenced by the orientation, aspect ratio, filler fraction and material of the fibers, with the primarily intention to confirm the reliability of equations 10-12. The FEM modelling strategy presented in this paper was an improved version of the model previously presented in Nilsson et al. [21, 24]. An overview of the simulation process is presented in Fig. 1.

The typical number of mesh elements for a composite comprising 30 fiber sections is about 250,000 tetrahedrals. Figs. 2 and 3 show typical meshes of a small model composite.

After constantly updating and improving in the past years, the simulation platform performs with high degree of flexibility, high degree of automation, high efficiency and high accuracy. Fig. 4 shows the mesh quality control of the simulation. Within the platform, all the factors could be controlled as either constant or variable, and a deeper understanding of the relationship between different factors is comprehended. An experimental consideration with multiple levels of variables is carried out, and the synergistic relationship 


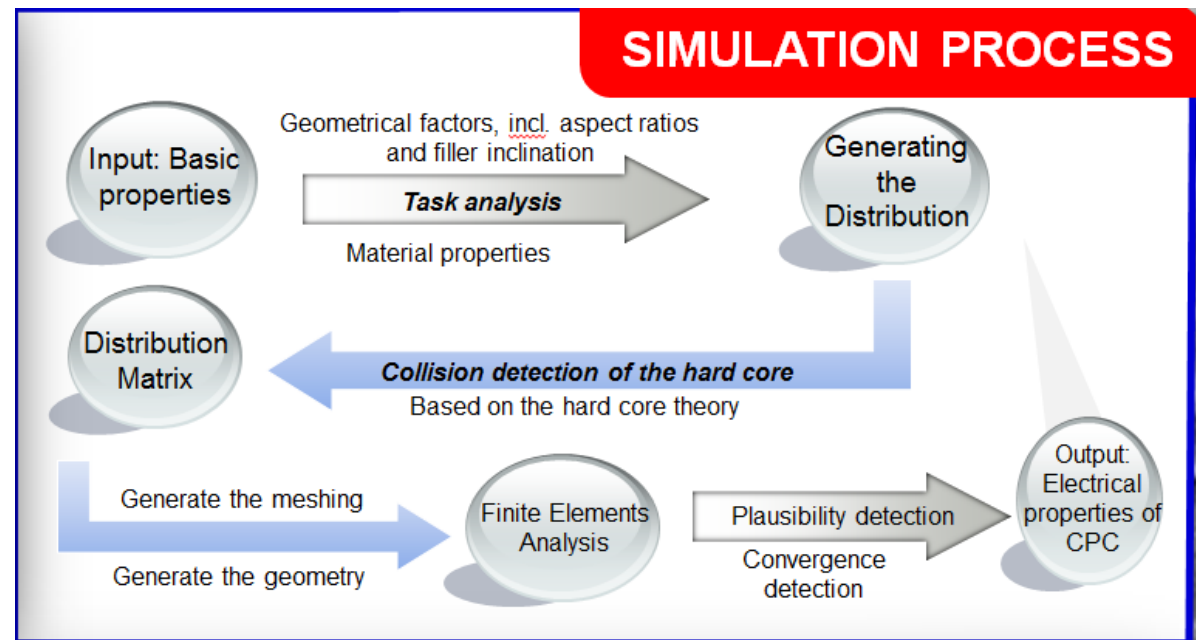

Fig. 1 Process of the simulation set in the platform.

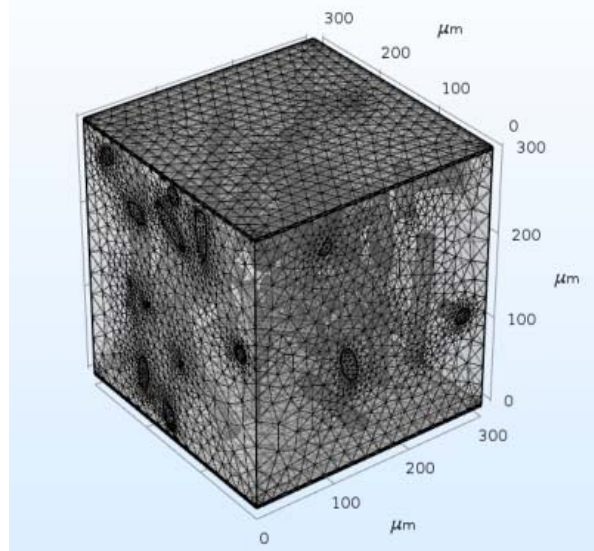

(a)

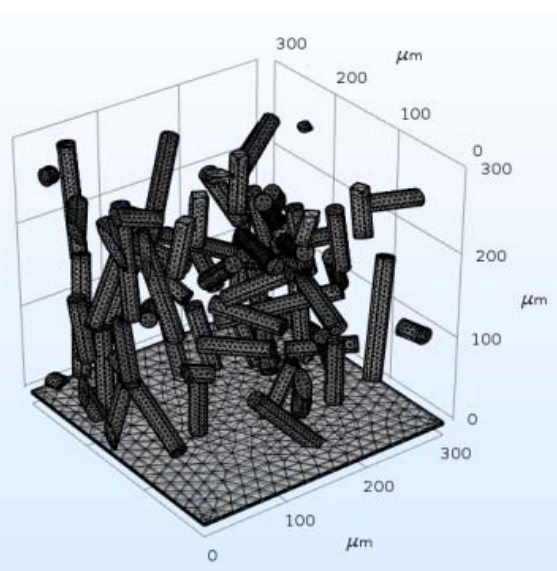

(b)

Fig. 2 (a) Mesh strategy for the simulation with $1 \sim 150$ fillers, with the top and bot-voltage blocks a coefficient of geometry scale 0.3. The coefficient of the space and the fillers is 1.0. The mesh operation over the geometry is set to be free tetrahedral. (b) Mesh of the conductive filler, with the top voltage block and the space set to be hidden.

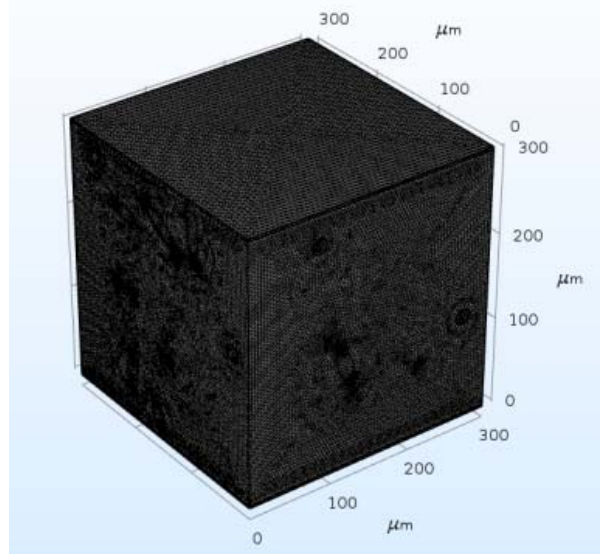

(a)

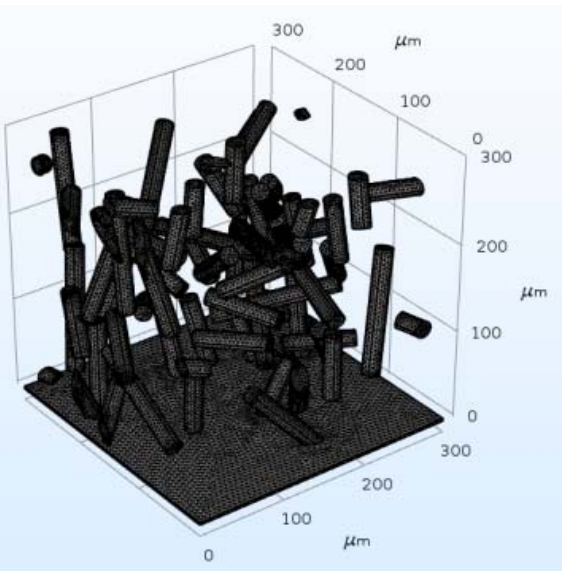

(b)

Fig. 3 (a) Mesh strategy for the simulation with $>150$ fillers, with the top and bot-voltage blocks a coefficient of geometry scale 2.0. The coefficient of the space and the fillers is 10.0. The mesh operation over the geometry is set to be free tetrahedral. (b) Mesh of the conductive filler, with the top voltage block and the space set to be hidden. 


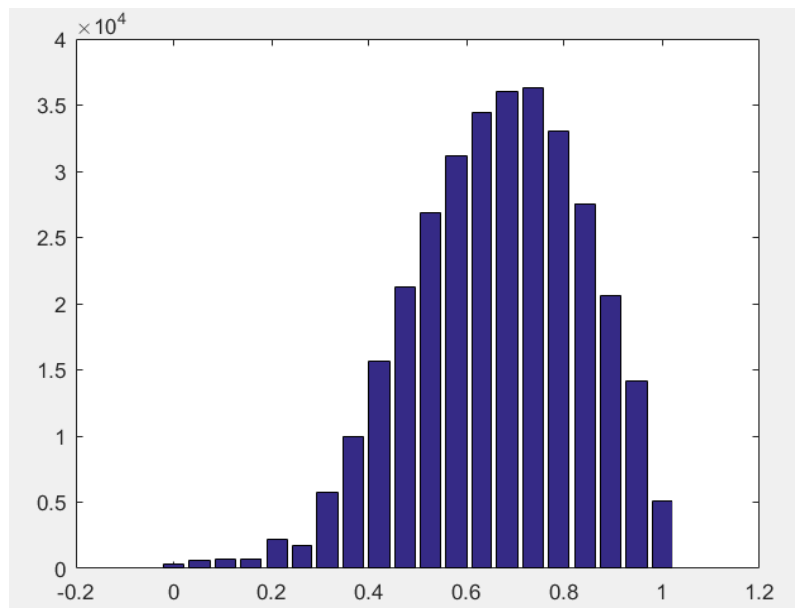

Fig. 4 Mesh quality control.

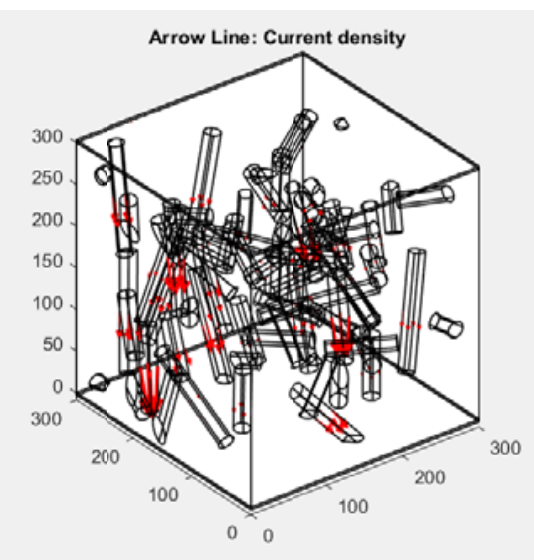

(a)

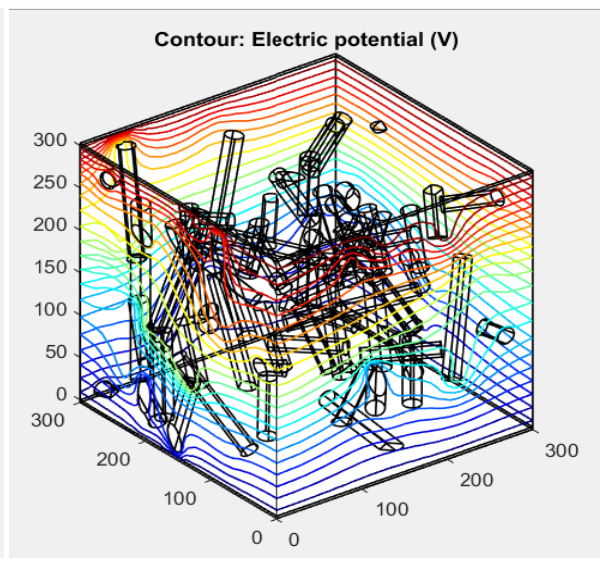

(b)

Fig. 5 Output of the current density and the electric potential.

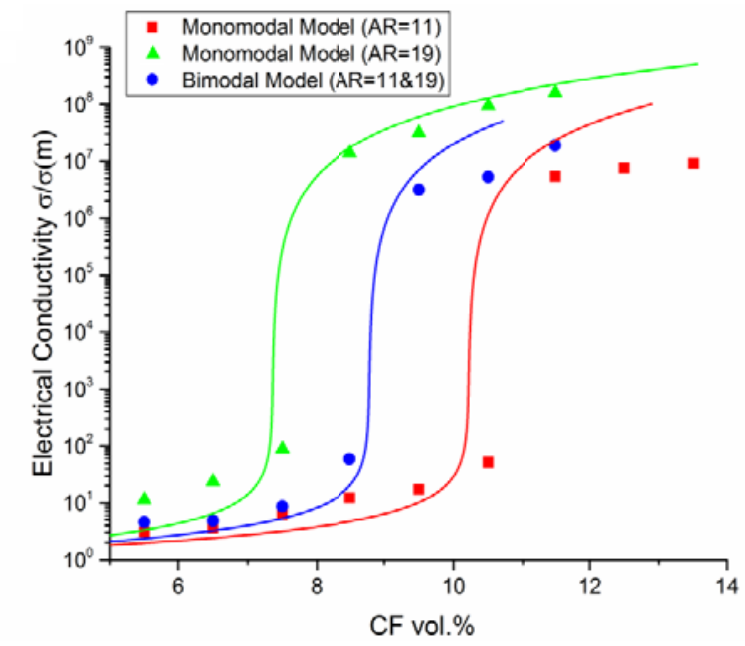

Fig. 6 Logarithm of conductivity vs. CF volume fraction for the composites from different aspect ratios, as well as the bimodal model. The filler fraction of the both kinds of CFs in the bimodal model is set to be $\mathbf{5 0 \%}$. With the change in composition, the conductivity of the CPCs as well as the percolation threshold will also show a corresponding change. of the variables could be systematic studied. The trend of change in each variable under different environmental settings is clearly displayed and systematically studied by the simulation platform.

In Fig. 5, the arrow line of current density shows automatically the path built by the conductive fillers, the denser the red arrows, the greater is the current flow, which also indicates the less resistance of the path. This output is significant at showing the electrical flow within the matrix according to the distribution of the fillers. After collecting more than 1,000 groups of data and calculating the median value out of it, a McLachlan Fit has been done according to the function.

Fig. 6 shows the McLachlan Fit of the data, and the mono modal with aspect ratio 19 has a lowest percolation threshold of $7.6 \%$; the group with $\mathrm{AR}=11$ 
has the highest percolation threshold of $10.2 \%$. With a bimodal model of a certain ratio between the both kinds of fillers (1:1), the percolation threshold could be adjusted to an intermediate value $8.4 \%$. With more data from the simulation platform, a preparation method with a fixed percolation threshold will be determined.

\section{Conclusions}

A significant research aiming at the difficulties of simulating CPCs is carried out as the first phase of the simulation. The platform will be systematically updated for a better performance and efficiency, especially the PTM (percolation threshold method) module and the "high conductive layer" feature. Through the whole working phase, all the factors with high research value will be controlled as either constant or variable, and a deeper understanding of the relationship between different factors will be comprehended. An experimental consideration with multiple levels of variables will be carried out, and the synergistic relationship will be systematically studied. Combined with experimental data, the trend of change in each variable under different environmental settings will be clearly displayed by the simulation platform.

\section{Acknowledgements}

The authors would like to acknowledge financial support of the Europa Akademie e.V. and Cetrico Industrial Consultant GmbH.

Dr. Monika Voigt is thanked for the instructive ideas and kind modifications (LSP, University of Erlangen-Nuremberg).

\section{References}

[1] Ram, R., Rahaman, M., Aldalbahi, A., and Khastgir, D. 2017. "Determination of Percolation Threshold and Electrical Conductivity of Polyvinylidene Fluoride (PVDF)/Short Carbon Fiber (SCF) Composites: Effect of SCF Aspect Ratio.” Polymer International 66: 573-82.

[2] Ondracek, G., and Schulz, B. 1973. "The Porosity Dependence of the Thermal Conductivity for Nuclear Fuels.” Journal of Nuclear Materials 46: 253-8.

[3] Niesel, W. 1952. "Die Dielektrizitätskonstanten heterogener Mischkörper aus isotropen und anisotropen Substanzen.” Annalen der Physik 445: 336-48.

[4] Ondracek, G. 1986. "Microstructure-Thermomechanical-Property

Correlations of Two-Phase and Porous Materials.” Materials Chemistry and Physics 15: 281-313.

[5] Nielsen, L. E. 1974. "The Thermal and Electrical Conductivity of Two-Phase Systems.” Industrial \& Engineering Chemistry Fundamentals13: 17-20.

[6] Bigg, D. M. 1977. "Conductive Polymeric Compositions.” Polymer Engineering \& Science 17: 842-7.

[7] Mamunya, E. P., Davidenko, V. V., and Lebedev, E. V. 1996. "Effect of Polymer-Filler Interface Interactions on Percolation Conductivity of Thermoplastics Filled with Carbon Black.” Composite Interfaces 4: 169-76.

[8] Weber, M., and Kamal, M. R. 1997. "Estimation of the Volume Resistivity of Electrically Conductive Composites.” Polymer Composites 18: 711-25.

[9] McLachlan, D. S., Blaszkiewicz, M., and Newnham, R. E. 1990. "Electrical Resistivity of Composites." Journal of the American Ceramic Society 73: 2187-203.

[10] Lux, F. 1993. "Models Proposed to Explain the Electrical Conductivity of Mixtures Made of Conductive and Insulating Materials.” Journal of Materials Science 28: 285-301.

[11] Kirkpatrick, S. 1973. "Percolation and Conduction." Reviews of Modern Physics 45: 574.

[12] Qu, M., and Schubert, D. W. 2016. "Conductivity of Melt Spun PMMA Composites with Aligned Carbon Fibers.” Composites Science and Technology 136: 111-8.

[13] Balberg, I., Anderson, C. H., Alexander, S., and Wagner, N. 1984. "Excluded Volume and Its Relation to the Onset of Percolation.” Physical Review B 30: 3933.

[14] Qu, M., Nilsson, F., Qin, Y., Yang, G., Pan, Y., Liu, X., Rodriguez, G. H., Chen, J., Zhang, C., and Schubert, D. W. 2017. "Electrical Conductivity and Mechanical Properties of Melt-Spun Ternary Composites Comprising PMMA, CARBON FIBers and Carbon Black." Composites Science and Technology 150: 24-31.

[15] Clingerman, M. L., King, J. A., Schulz, K. H., Meyers, J. D. 2002. "Evaluation of Electrical Conductivity Models for Conductive Polymer Composites.” Journal of Applied Polymer Science 83: 1341-56.

[16] Radzuan, N. A. M., Sulong, A. B., and Sahari. J. A. 2017. "Review of Electrical Conductivity Models for Conductive Polymer Composite.” International Journal of Hydrogen Energy 42: 9262-73.

[17] Mamunya, Y. P., Davidenko, V. V., Pissis, P., and Lebedev, E. V. 2002. "Electrical and Thermal Conductivity of Polymers Filled with Metal Powders.” European Polymer Journal 38: 1887-97.

[18] Dalmas, F., Dendievel, R., Chazeau, L., Cavaillé, J.-Y., 

Electrical Properties of Conductive Polymer Composites

and Gauthier, C. 2006. "Carbon Nanotube-Filled Polymer Composites. Numerical Simulation of Electrical Conductivity in Three-Dimensional Entangled Fibrous Networks.” Acta materialia 54: 2923-31.

[19] Starý, Z., Krückel, J., Weck, C., and Schubert, D. W. 2013. "Rheology and Conductivity of Carbon Fibre Composites with Defined Fibre Lengths.” Composites Science and Technology 85: 58-64.

[20] Krückel, J., Starý, Z., Triebel, C., Schubert, D. W., and Münstedt, H. 2012. "Conductivity of Polymethylmethacrylate Filled with Carbon Black or Carbon Fibres under Oscillatory Shear." Polymer 53: 395-402.

[21] Nilsson, F., Gedde, U. W., and Hedenqvist, M. S. 2011. "Modelling the Relative Permittivity of Anisotropic Insulating Composites." Composites Science and
Technology 71 (2): 216-21.

[22] Nilsson, F., and Unge, M. 2016. "Conductivity Simulations of Field-Grading Composites." Journal of Physics D: Applied Physics 49 (33): 335303.

[23] Yi, J. Y., and Choi, G. M. 1999. "Percolation Behavior of Conductor-Insulator Composites with Varying Aspect Ratio of Conductive Fiber.” Journal of Electroceramics 3: 361-9.

[24] Nilsson, F., Krueckel, J., Schubert, D. W., Chen, F., Unge, M., Gedde, U. W., and Hedenqvist, M. S. 2016. "Simulating the Effective Electric Conductivity of Polymer Composites with High Aspect Ratio Fillers.” Composites Science and Technology 132: 16-23.

[25] McLachlan, D. S., and Sauti, G. 2007. "The AC and DC Conductivity of Nanocomposites.” Journal of Nanomaterials 2007: 15. 